\title{
DOUBLY-CONNECTED MINIMAL SURFACES $\left({ }^{1}\right)$
}

\author{
BY \\ PAUL M. BAILYN
}

1. Introduction. Summary of results. The purpose of this paper is to establish necessary conditions and sufficient conditions for two curves in three-dimensional euclidean space to bound a doubly-connected minimal surface. Loosely stated, it is shown that if the two curves are to bound a doubly-connected minimal surface then it is necessary that they not be far apart relative to their diameters and it is sufficient that they be close to each other in the sense that one be a small perturbation of the other.

Results are obtained also for minimal surfaces of topological type other than doubly-connected.

Necessary conditions. Soap film experiments and analysis of the classical case of minimal surfaces of revolution (see [1]) make plausible the conjecture that as the two boundary curves of a doubly-connected minimal surface are pulled apart, a position is always reached beyond which no doubly-connected minimal surface spanning the curves exists.

One obtains such critical positions if the curves do not grow indefinitely in diameter and regardless of whether or not the shapes of the curves change. If the curve diameters are allowed to grow indefinitely then such a critical position may not occur as can be seen by the case of two separating coaxial circles obtained by taking sections of a minimal surface of revolution by separating planes perpendicular to the axis of the surface.

In connection with this J. C. C. Nitsche [7] proves that if the Jordan curves $\gamma_{1}$ and $\gamma_{2}$ bound a doubly-connected minimal surface then $d\left(\gamma_{1}, \gamma_{2}\right) \leqq 3 / 2 \max \left(d_{1}, d_{2}\right)$ where $d\left(\gamma_{1}, \gamma_{2}\right)$ is the distance between $\gamma_{1}$ and $\gamma_{2}$ and $d_{1}$ and $d_{2}$ are the diameters of $\gamma_{1}$ and $\gamma_{2}$, respectively.

If the curves $\gamma_{1}$ and $\gamma_{2}$ lie in parallel planes, Nitsche [6] obtains a somewhat stronger result: Let $\gamma_{1}$ lie in $z=c_{1}$ and $\gamma_{2}$ in $z=c_{2}\left(c_{1}<c_{2}\right)$, respectively. Chose a point $p_{1}=\left(x_{1}, y_{1}, c_{1}\right)$ in the plane $z=c_{1}$, in some sense the center of $\gamma_{1}$, and a point $p_{2}=\left(x_{2}, y_{2}, c_{2}\right)$ in the plane $z=c_{2}$, in some sense the center of $\gamma_{2}$. Let $r=c_{2}-c_{1}$ and $d=\left[\left(x_{2}-x_{1}\right)^{2}+\left(y_{2}-y_{1}\right)^{2}\right]^{1 / 2}$. Denote by $\delta_{1}$ the maximal distance of the point $p_{1}$ from the curve $\gamma_{1}$ and by $\delta_{2}$ the maximal distance of the point $p_{2}$ from the curve $\gamma_{2}$. Nitsche's theorem then states that if $\gamma_{1}$ and $\gamma_{2}$ bound a doubly-connected minimal surface then $\left(r^{2}+\frac{1}{2} d^{2}\right)^{1 / 2} \leqq \delta_{1}+\delta_{2}$.

Received by the editors January 24, 1966.

${ }^{(1)}$ This paper forms part of the author's doctoral thesis under the direction of Professor Richard Courant at the Courant Institute of Mathematical Sciences, New York University, New York. The research was supported by the National Science Foundation. 
Theorem 2.1 of this paper also deals with curves in parallel planes and compares doubly-connected minimal surfaces in general with minimal surfaces of revolution (special catenoids): Let $\gamma_{1}, \gamma_{2}$ be two Jordan curves in three-dimensional euclidean space lying in parallel planes and suppose there is a doubly-connected minimal surface $G$ spanning $\gamma_{1}, \gamma_{2}$. Then if $C_{1}, C_{2}$ are coaxial circles lying in the planes of $\gamma_{1}, \gamma_{2}$ and enclosing $\gamma_{1}, \gamma_{2}$, respectively, we can conclude that there is a minimal surface of revolution spanning $C_{1}, C_{2}$ which encloses $G$.

On the basis of this theorem it is then shown, as Theorem 2.2, that the classical case of doubly-connected minimal surfaces of revolution constitutes a limiting case in which boundary curves of a doubly-connected minimal surface are pulled apart as far as the existence of a doubly-connected minimal surface permits: Let $\gamma_{1}, \gamma_{2}$ be two Jordan curves in three-dimensional euclidean space contained in a right circular cylinder of unit diameter and separated by each of two planes $\Pi_{1}, \Pi_{2}$. $\Pi_{1}$ and $\Pi_{2}$ are each to be perpendicular to the axis of the cylinder and the distance between them we denote by $M$.

We now pose the problem of finding sup $M$, where $\gamma_{1}, \gamma_{2}$ satisfy the above requirements and in addition are to bound a doubly-connected minimal surface.

The solution is given by $\sup M=M_{0}=0.6627 \ldots$ where $M_{0}=0.6627 \ldots$ is the maximum distance between coaxial circles of unit diameter for which a nondegenerate minimal surface of revolution spans them.

The result is sharp since this classical case of minimal surfaces of revolution realizes the supremum.

Another interesting necessary condition introduced in this paper is the result (Theorem 2.3) that the curves $\gamma_{1}, \gamma_{2}$ do not bound a doubly-connected minimal surface if the projections of $\gamma_{1}, \gamma_{2}$ on some plane are far enough apartthe curves themselves may have large, even infinite, diameters: Let $\gamma_{1}, \gamma_{2}$ be two Jordan curves in three-dimensional euclidean space with the property that there exists a plane $\Pi, x y$-axes in $\Pi$ and a positive number $c$ such that the respective projections $\gamma_{1}^{\prime}, \gamma_{2}^{\prime}$ of $\gamma_{1}, \gamma_{2}$ onto $\Pi$ lie in the regions $y>c \cosh (x / c)$ and $y<-c \cosh (x / c)$, respectively. Then $\gamma_{1}, \gamma_{2}$ do not bound a doubly-connected minimal surface.

Geometric sufficient conditions. Using his general sufficient condition for existence of minimal surfaces of arbitrarily specified topological type J. Douglas was, in particular, able to establish existence of doubly-connected minimal surfaces for interlacing curves in $R^{3}$ and for certain curves lying in parallel planes. (See [4].) In this paper, using the sufficient condition of Douglas, we establish another geometric condition, (Theorem 3.1) on the curves $\gamma_{1}, \gamma_{2}$ which ensures the existence of a doubly-connected minimal surface spanning the curves: Let $\gamma$ be a rectifiable Jordan curve of length $L$ in $R^{3}$ and $\eta(\varepsilon)>0$ a function of $\varepsilon>0$ which tends to zero with $\varepsilon$. Then there exists an $\varepsilon_{0}>0$ with the property that whenever $0<\varepsilon \leqq \varepsilon_{0}$, any two Jordan curves $\gamma_{1}, \gamma_{2}$ simply-threading $T_{\varepsilon}(\gamma)$ with lengths $L_{1}, L_{2}$, respectively, satisfying $L_{i} \leqq L+\eta(\varepsilon),(i=1,2)$, bound a doubly-connected minimal surface $G$. 
$G$ has area (strictly) smaller than the sum of the minima of areas of disk-like surfaces spanning $\gamma_{1}$ and $\gamma_{2}$ individually.

For the definition of "tube $T_{\varepsilon}(\gamma)$ " and other terms see subsection 3.1, Preliminary material.

Theorem 3.1 can then be modified slightly to yield a theorem (Theorem 3.2) dealing with the existence of minimal surfaces of the Möbius type: Let $\gamma$ be $a$ closed, rectifiable Jordan curve of length $L$ in $R^{3}$ and $\eta(\varepsilon)>0$ a function of $\varepsilon>0$ which tends to zero with $\varepsilon$. Then there exists an $\varepsilon_{0}>0$ with the property that whenever $0<\varepsilon \leqq \varepsilon_{0}$ any Jordan curve $\gamma^{\prime}$ doubly-threading $T_{\varepsilon}(\gamma)$ with length $L^{\prime}$ satisfying $L^{\prime} \leqq 2 L+\eta(\varepsilon)$ bounds a minimal surface $G$ of the Möbius type. $G$ has area (strictly) smaller than the g.l.b. of areas of disk-like surfaces spanning $\gamma^{\prime}$.

In $\$ 4$ examples are given illustrating the results summarized above.

2. Necessary conditions. In this section we will establish the results stated in the introduction concerning the necessity that two curves bounding a doublyconnected minimal surface be close in the senses described in the statements of the theorems.

2.1. Two lemmas. The lemmas established in this paragraph will be made use of in the proofs of theorems in subsections $2.2,2.3$, and 2.4.

Lemma 2.1. Let the two surfaces $\Sigma, G$ be tangent at the point $P$ with $G$ lying completely on one side of $\Sigma$. Let the principal curvatures on $\Sigma$ at $P$ be $k_{1}, k_{2}$ with $k_{1}>0 . k_{1}>0$ indicates that the corresponding principal curve on $\Sigma$ at $P$ is concave toward $G$. Then if $k_{2}>-k_{1}, G$ cannot have mean curvature zero at $P$, so that $G$ cannot be a minimal surface.

Proof. We choose the cartesian $x y z$-system so that the normal to $\Sigma$ at $P$ lies along the $z$-axis with the $x$-and $y$-axes tangent to the principal curves with principal curvatures $k_{1}$ and $k_{2}$, respectively.

Then $\Sigma$, in some neighborhood of $P$, is given by $z_{\Sigma}=k_{1} x^{2}+k_{2} y^{2}+o\left(r^{2}\right)$ where $r^{2}=x^{2}+y^{2}$ and $\lim _{r \rightarrow 0} o\left(r^{2}\right) / r^{2}=0$.

Let us assume that $G$ has mean curvature zero at $P$; it can then be expressed as follows in some neighborhood of $P: z_{G}=k x^{2}+k^{\prime} x y-k y^{2}+o\left(r^{2}\right)$.

From the hypothesis we have $z_{G} \geqq z_{\Sigma}$ in some neighborhood of $P$. Hence, there is a neighborhood of $P$ in which $\left(k_{1}-k\right) x^{2}-k^{\prime} x y\left(k_{2}+k\right) y^{2}+o\left(r^{2}\right) \leqq 0$. In this last relation let $x=0, r=y$; then $\left(k_{2}+k\right)+o\left(r^{2}\right) / r^{2} \leqq 0$. Letting $r \rightarrow 0$ we obtain $k \leqq-k_{2}<k_{1}$, since $k_{2}>-k_{1}$.

Next, let $y=0, r=x$ and $r \rightarrow 0$; this yields $k \geqq k_{1}$, in contradiction to $k<k_{1}$. Thus $G$ cannot have mean curvature zero at $P$ and Lemma 2.1 is proved.

Lemma 2.2. Suppose that $\gamma_{1}, \gamma_{2}$ are two curves in three-dimensional euclidean space separated by, and with no points in common with, each of two parallel planes $\Pi_{1}$ and $\Pi_{2}$, and $G$ a doubly-connected minimal surface spanning $\gamma_{1}, \gamma_{2}$. Then $\Pi_{1}$ 
and $\Pi_{2}$ intersect $G$ in two curves $\gamma_{1}^{*}, \gamma_{2}^{*}$, respectively with $\gamma_{1}^{*}, \gamma_{2}^{*}$ bounding a portion of $G$ which itself is a doubly-connected minimal surface.

Proof. Suppose that $G$ in $x y z$-space is the image by the harmonic vector $x(u, v)$ of the circular ring $B: 0<a \leqq r=\left(u^{2}+v^{2}\right)^{1 / 2} \leqq 1$ in the parameter $u v$-plane with the image of $\beta_{1}: r=1$, being $\gamma_{1}$ and the image of $\beta_{2}: r=a$, being $\gamma_{2}$.

To show that $\gamma_{1}^{*}, \gamma_{2}^{*}$ exist we take the cartesian coordinates $x=(x, y, z)$ with the $z$-axis perpendicular to $\Pi_{1}$ and $\Pi_{2}$. Then $z(u, v)$ is harmonic and $z(u, v)=$ const $=c_{i}$ on $F_{i}$ where $F_{i}$ is the pre-image under $\boldsymbol{x}(u, v)$ of the intersection of $G$ with $\Pi_{i}$. From the theory of harmonic functions the point set $F_{i}$ consists of analytic arcs with possible singularities occurring only at crossing points. But if there were a crossing point one analytic arc would necessarily reach the boundary $\beta_{1} \cup \beta_{2}$. This, however, would mean that a point of the boundary $\beta_{1} \cup \beta_{2}$ maps into $\gamma_{1}^{*} \cup \gamma_{2}^{*}$. This is impossible since the planes $\Pi_{i}$ do not touch the curves $\gamma_{i}$. Hence $F_{i}$ must be an analytic curve which is bounded away from both $\beta_{1}$ and $\beta_{2}$.

Since $\Pi_{1}$ and $\Pi_{2}$ are disjoint we also have that $F_{1}$ and $F_{2}$ are disjoint. Finally, any continuous curve $\lambda$ joining any point of $\beta_{2}$ to any point of $\beta_{1}$ must intersect $F_{1}$ and $F_{2}$; any $\lambda$ joining $\beta_{2}$ to $F_{1}$, or $F_{2}$ to $\beta_{1}$ must intersect $F_{2}$ or $F_{1}$, respectively.

These observations allow us to conclude that $F_{1}$ and $F_{2}$ are closed curves homologous to each other and to $\beta_{1}$ and $\beta_{2}$ in $B$. Also $\beta_{1}, F_{1}, F_{2}, \beta_{2}$ are disjoint and occur in that order.

We define $\gamma_{i}^{*}$ by $\gamma_{i}^{*}=\boldsymbol{x}\left(F_{i}\right)$ and easily see that the $\gamma_{i}^{*}$ have the properties required.

2.2. Curves in parallel planes. Comparison with catenoids. This paragraph is devoted to the proof of

THeOREM 2.1. Let $\gamma_{1}, \gamma_{2}$ be two Jordan curves in three-dimensional euclidean space lying in parallel planes and suppose that there is a doubly-connected minimal surface $G$ spanning $\gamma_{1}, \gamma_{2}$. Then if $C_{1}, C_{2}$ are coaxial circles lying in the planes of $\gamma_{1}, \gamma_{2}$ and enclosing $\gamma_{1}, \gamma_{2}$, respectively, we can conclude that there is a minimal surface of revolution spanning $C_{1}, C_{2}$ which encloses $G$.

Proof. The proof of this theorem is based on Lemma 2.1. We construct a set of saddle surfaces of revolution $\left\{B_{s}\right\}$ bounded by $C_{1}$ and $C_{2}$ for which the inside principal curvature at any point is larger than the outside principal curvature at that point for any $B_{s}$.

As $s$ varies over its index set, $s_{2}<s \leqq s_{1}, B_{s}$ will be continuously deformed from $B_{s_{1}}$, a right circular cylinder, to $B_{s_{2}}$.

In the case that $C_{1}, C_{2}$ bound a minimal surface $\left({ }^{2}\right)$ of revolution, $B_{s_{2}}$ will be the stable one (i.e., the one closest to $B_{s_{1}}$ ).

If $C_{1}, C_{2}$ bound a unique minimal surface of revolution then $B_{s_{2}}$ is that surface.

Finally, if $C_{1}$ and $C_{2}$ are so far apart that no minimal surface of revolution

(2) Unless otherwise specified we shall always consider nondegenerate (connected) minimal surfaces. 
spans them, $B_{s_{2}}$ will be a degenerate surface of revolution, consisting of two plane disks and a line joining their centers.

With the aid of Lemma 2.1, once the existence of the $B_{s}$ are established, we proceed as follows: $B_{s_{1}}$ must contain any minimal surface $G$ spanning $\gamma_{1}, \gamma_{2}$ since $G$ is contained in the convex hull of $\gamma_{1}$ and $\gamma_{2}$, which in turn is contained in the convex hull of $C_{1}$ and $C_{2}$, i.e., $B_{s_{1}}$.

We now let $s$ decrease from $s_{1}$ supposing $B_{s_{2}}$ to be nondengerate. If $B_{s}$ ever touches $G$ at a point not on $\gamma_{1}$ or $\gamma_{2}$ there must be a first such point of contact $P$. At $P, B_{s}$ is tangent to $G$ and Lemma $2.1\left(^{3}\right)$ applies with $\Sigma=B_{s}$. Hence, for this $s=s^{\prime}, B_{s^{\prime}}$ must be a minimal surface of revolution, i.e., $B_{s^{\prime}}=B_{s_{2}}$.

If $B_{s_{2}}$ is degenerate and $s$ decreases till $s=s_{2}$ with $B_{s}$ never touching $G$ for $s_{2}<s \leqq s_{1}$ then $G$ must be contained by a degenerate surface of revolution, $B_{s_{2}}$, and, hence, must be degenerate itself, contrary to hypothesis. Hence, there cannot be a connected $G$ for which $B_{s_{2}}$ is degenerate.

Consequently, $C_{1}, C_{2}$ must span a minimal surface of revolution which contains $G$ if we can prove the existence of the $B_{s}$. This will now be done.

Construction of the $B_{s}$. We take the distance between $C_{1}$ and $C_{2}$ to be $M$ and the radius of $C_{i}$ to be $a$. For the generators of $B_{s}$ we take catenaries:

$$
y=y(x ; s)=s \cosh (x / c), \quad c=c(s)>0 .
$$

We impose the condition $y(M / 2 ; s)=a=s \cosh (M / 2 c)$; with $a, M$ constants. This gives the relation $c=c(s)$ implicitly.

Since the curves of principal curvature on $B_{s}$ are meridian circles and the generators we can compute the principal curvatures $k_{1}(x), k_{2}(x)$ as follows: (Here $k_{2}(x)$ is the outside curvature and $k_{1}(x)$ the inside curvature at any point $P$ of $B_{s}$, on a meridian circle corresponding to the abscissa value $x$.)

$$
k_{2}(x)=y^{\prime \prime} /\left(1+y^{\prime 2}\right)^{3 / 2},
$$

with $y^{\prime}=(s / c) \sinh (x / c), y^{\prime \prime}=\left(s / c^{2}\right) \cosh (x / c)$.

For the inside principal curvature $k_{1}(x)$ we project the curvature vector of the meridian circle onto the surface normal at $P$.

Hence,

$$
k_{1}(x)=1 /\left(y\left(1+y^{\prime 2}\right)^{1 / 2}\right) .
$$

where $h=h(s)=s / c(s)$.

$$
\begin{aligned}
k_{2}(x) / k_{1}(x) & =-y y^{\prime \prime} /\left(1+y^{\prime 2}\right) \\
& =-h^{2} \cosh ^{2}(x / c) /\left(1+h^{2} \sinh ^{2}(x / c)\right),
\end{aligned}
$$

It is clear then that a necessary and sufficient condition for $B_{s}$ to be a minimal surface is that $s=c(s)$ since in this case and this case only will $k_{2}(x) / k_{1}(x)=-1$.

$\left({ }^{3}\right)$ For Lemma 2.1 to apply $G$ must be free of branch points or else the point of first contact $P$ on $G$ might be a branch point and the proof of Lemma 2.1 invalid. It is true, however, that doubly-connected minimal surfaces with boundary curves in parallel planes are free of branch points. For a proof of this see [6, p. 661]. 
Also, as $s$ decreases from $s=a=s_{1}$, where $B_{s_{1}}$ is a right circular cylinder, $B_{s}$ is always a valid comparison surface until $s$ reaches a value $s=s_{2}$ for which $s=c(s)$. This follows from the facts that $c(a)=+\infty$ and $h(s)$ is a continuous function of $s$. Thus, for $s_{2}<s \leqq s_{1}, h<1$ and

$$
\left|k_{2}(x) / k_{1}(x)\right|=h^{2} \cosh ^{2}(x / c) /\left(1+h^{2} \sinh ^{2}(x / c)\right)<1,
$$

the condition needed to show that $B_{s}$ is a comparison surface.

In the event that $s_{2}>0$ we have the case that $C_{1}, C_{2}$ bound at least one minimal surface of revolution. If $s_{2}=0$ then $C_{1}, C_{2}$ do not bound a (connected) minimal surface of revolution and $B_{s_{2}}$ is a degenerate surface consisting of the disks spanning $C_{1}$ and $C_{2}$ with the straight line joining their centers.

We have thus proved the existence of the set $\left\{B_{s}\right\}$ and with it Theorem 2.1.

2.3. The main theorem. Classical case as solution of variational problem. We are now in a position to prove the main result of this section, that the classical case of doubly-connected minimal surfaces of revolution (special catenoids) constitutes a limiting case in which boundary curves of a doubly-connected minimal surface are pulled as far apart as the existence of a doubly-connected minimal surface permits. Precisely, we have

THEOREM 2.2. Let $\gamma_{1}, \gamma_{2}$ be two Jordan curves in three-dimensional euclidean space contained in a right circular cylinder of unit diameter and separated by each of two planes $\Pi_{1}, \Pi_{2} . \Pi_{1}$ and $\Pi_{2}$ are each to be perpendicular to the axis of the cylinder and the distance between them we denote by $M$.

We now pose the problem of finding sup $M$, where $\gamma_{1}, \gamma_{2}$ satisfy the above requirements and in addition are to bound a doubly-connected minimal surface.

The solution is given by $\sup M=M_{0}=0.6627 \ldots$ where $M_{0}=0.6627 \ldots$ is the maximum distance between coaxial circles of unit diameter for which a nondegenerate minimal surface of revolution spans them.

The result is sharp since this classical case of minimal surfaces of revolution realizes the supremum.

Proof. Suppose that sup $M>M_{0}$; then there exists two curves $\gamma_{1}^{\prime}, \gamma_{2}^{\prime}$ in the cylinder bounding a doubly-connected minimal surface, separated by planes $\Pi_{1}^{\prime}, \Pi_{2}^{\prime}$ each perpendicular to the axis of the cylinder and separated by distance $M^{\prime}>M_{0}$.

Applying Lemma 2.2 we obtain curves $\gamma_{1}^{\prime \prime}, \gamma_{2}^{\prime \prime}$ lying, respectively, in the planes $\Pi_{1}^{\prime}, \Pi_{2}^{\prime}$ and bounding a doubly-connected minimal surface. With $C_{1}, C_{2}$ denoting the circles which are the intersections of $\Pi_{1}^{\prime}, \Pi_{2}^{\prime}$ with the cylinder we apply Theorem 2.1 and conclude that $C_{1}, C_{2}$ bound a minimal surface of revolution. However, this is impossible since $M_{0}$ is the largest such value of $M$ for which this can occur. Hence, the theorem is proved.

We note further that the class of admissible curves $\gamma_{1}, \gamma_{2}$ allowed for competition in the variational problem $M=$ sup can easily be extended to include those curves 
which have more than one component and which bound minimal surfaces of arbitrary topological structure although we must here stipulate that they be free of branch points. In this more general case Lemma 2.1 can still be used in a proof using the comparison surfaces $B_{s}$ of paragraph 2.2. One need not deal with the plane case first, as in Theorem 2.1 but, instead, prove the generalized theorem directly.

2.4. Close curves with large diameters. We here prove the interesting result that the curves $\gamma_{1}, \gamma_{2}$ do not bound a doubly-connected minimal surface if the projections of $\gamma_{1}, \gamma_{2}$ on some plane are far enough apart-the curves themselves may have large, even infinite, diameters. Precisely, we prove

THEOREM 2.3. Let $\gamma_{1}, \gamma_{2}$ be two Jordan curves in three-dimensional euclidean space with the property that there exists a plane $\Pi$, xy-axes in $\Pi$ and a positive number $c$ such that the respective projections $\gamma_{1}^{\prime}, \gamma_{2}^{\prime}$ of $\gamma_{1}, \gamma_{2}$ onto $\Pi$ lie in the regions $y>c \cosh (x / c)$ and $y<-c \cosh (x / c)$, respectively. Then $\gamma_{1}, \gamma_{2}$ do not bound $a$ doubly-connected minimal surface.

Proof. Suppose some $\gamma_{1}, \gamma_{2}$ satisfying the hypothesis of the theorem, do bound a doubly-connected minimal surface $G$. With $\varepsilon>0$ so small that $y= \pm(c+\varepsilon) \cosh (x / c)$ do not touch $\gamma_{1}^{\prime}$ or $\gamma_{2}^{\prime}$ we construct a surface of revolution $B$ by rotating $y=(c+\varepsilon) \cosh (x / c)$ about the $x$-axis. $B$ has outside principal curvature greater than inside principal curvature $\left(\left|k_{2}(x) / k_{1}(x)\right|=h^{2} \cosh ^{2}(x / c) /\left(1+h^{2} \sinh ^{2}(x / c)\right)>1\right.$ since $h=(c+\varepsilon) / c>1)$ and hence is a valid comparison surface with respect to surfaces on the outside of $B$ in the sense of Lemma 2.1 .

We now translate $B$ in a direction perpendicular to $\Pi$ until $G$ is outside $B$. (For example, until $B$ has no points of contact with the convex hull of $\gamma_{1}$ and $\gamma_{2}$.) From this position we translate $B$ toward $\Pi$ noting that by the reasoning of 2.2 (using Lemma 2.1) $B$ can never touch $G$. Continuing to translate $B$ indefinitely in this manner we see that $G$ could never have existed in the first place. The theorem is proved.

Notes: (1) The theorem remains true if $\gamma_{1}$ and/or $\gamma_{2}$ extend to infinity in a direction perpendicular to $\Pi$ without a modification of the proof.

(2) No (branch point free) minimal surface (regardless of topological type) can span $\gamma_{1}, \gamma_{2} ; \gamma_{1}, \gamma_{2}$ consisting, perhaps, of more than one component and still satisfying the hypotheses of Theorem 2.3. See the comment at the end of paragraph 2.3.

As a simpler necessary criterion than that of Theorem 2.3 we offer

COROLlaRY 2.1. Let $\gamma_{1}, \gamma_{2}$ be two Jordan curves in three-dimensional euclidean space with the property that there exists a plane $\Pi$ on which the distance $d\left(\gamma_{1}^{\prime}, \gamma_{2}^{\prime}\right)$ between the projections $\gamma_{1}^{\prime}, \gamma_{2}^{\prime}$ of $\gamma_{1}, \gamma_{2}$ satisfies

$$
d\left(\gamma_{1}^{\prime}, \gamma_{2}^{\prime}\right)>\alpha \max \left(d_{1}, d_{2}\right)=1.574 \ldots \max \left(d_{1}, d_{2}\right)
$$

where $d_{i}$ is the diameter of $\gamma_{i}^{\prime}$ and $\alpha=(\cosh k) / k$, with $k \tanh k=1,(\alpha=1.574 \ldots)$. Then $\gamma_{1}, \gamma_{2}$ do not bound a doubly-connected minimal surface. 
Proof. It is a simple matter to show that if $\gamma_{1}^{\prime}, \gamma_{2}^{\prime}$ satisfy the conditions of the corollary then $x y$-axes can be introduced in $\Pi$ and an appropriate $c>0$ found such that Theorem 2.3 applies.

The two notes after the proof of Theorem 2.3 apply here as well.

3. Geometric sufficient conditions. In this section we are concerned with geometric sufficient conditions for the existence of doubly-connected and Möbius type minimal surfaces. The basis of the discussion will be the sufficient condition of Douglas: A system of Jordan curves $\gamma_{1}, \ldots, \gamma_{v}$ will bound a minimal surface $G$ of some prescribed topological type if the g.l.b. of areas of surfaces spanning $\gamma_{1}, \ldots, \gamma_{v}$ of the prescribed type is (strictly) less than the g.l.b., $\sigma$, of areas of all surfaces of lower type. If this is the case then the area $A(G)$ of $G$ satisfies: $A(G)<\sigma$. See [5].

3.1. Preliminary material. Before proceeding to the statements and proofs of the geometric conditions we present some preliminary material.

Let $\gamma$ be a closed rectifiable Jordan curve in $R^{3}$ with parametric representation $\boldsymbol{x}=\boldsymbol{g}(\theta), 0 \leqq \theta<2 \pi$. If $\varepsilon$ is a positive number we define the tube $T_{\varepsilon}(\gamma)$ with centerline $\gamma$ and radius $\varepsilon$ as the union of all spheres of radius $\varepsilon$ with center on $\gamma$. A point $P$ is in $T_{\varepsilon}(\gamma)$ if and only if there is a point $Q \in \gamma$ for which the distance between $P$ and $Q, d(P, Q) \leqq \varepsilon$. A point set is in $T_{\varepsilon}(\gamma)$ if each point is in $T_{\varepsilon}(\gamma)$.

Consider the curve $\gamma^{(n)}$ with parametric representation $x=g_{n}(\theta)=g(n \theta), 0 \leqq \theta<2 \pi$. $\gamma^{(n)}$ can be considered as $\gamma$ traversed $n$ times; e.g., $\gamma=\gamma^{(1)}$.

By the curve $\gamma^{\prime}, n$-fold threading $T_{\varepsilon}(\gamma)$ we mean that $\gamma^{\prime}$ can be obtained from $\gamma^{(n)}$ by means of a continuous deformation such that at each stage of the deformation the curve is in $T_{\varepsilon}(\gamma)$. In particular $\gamma^{\prime}$ is in $T_{\varepsilon}(\gamma)$. If $n=1$ we say that $\gamma^{\prime}$ simply-threads $T_{\varepsilon}(\gamma) . \gamma^{\prime}$ is said to have the same orientation as $\gamma$ if under the continuous deformation just mentioned orientation varies continuously.

Note that although every point $P$ of $\gamma^{\prime} n$-fold threading $T_{\varepsilon}(\gamma)$ is within $\varepsilon$ distance of a point $Q$ of $\gamma$, it is not true that every point $Q$ of $\gamma$ is within $\varepsilon$ of a point $P$ on $\gamma^{\prime}$. We can, however, prove the following lemma.

LeMma 3.1. For all $\mu>0$ there exists an $\varepsilon>0$ such that for any point $Q \in \gamma$ and curve $\gamma^{\prime} n$-fold threading $T_{\varepsilon}(\gamma)$ we have $d\left(Q, \gamma^{\prime}\right) \leqq \mu$.

Proof. Since $\gamma$ is a closed rectifiable Jordan curve in $R^{3}$ we can find another closed rectifiable Jordan curve $\gamma^{*}$ in $R^{3}$ "interlocking" with $\gamma$. That is, if $\gamma$ is continuously deformed into a point, at some stage of the deformation the curve must interest $\gamma^{*}$.

Let the distance $d\left(\gamma, \gamma^{*}\right)$ between $\gamma$ and $\gamma^{*}$ be $d>0$. Consider only those values of $\varepsilon$ for which $\varepsilon<d$. Let us suppose now that there is a point $Q_{0}$ of $\gamma$ and a positive number $\mu_{0}$ such that no matter how small $\varepsilon$ is, $d\left(Q_{0}, \gamma^{\prime}\right) \geqq \mu_{0}$. Then as $\varepsilon \rightarrow 0$ the set of limit points of the $\gamma^{\prime}$, which necessarily lie on $\gamma$, does not contain $Q_{0}$.

Since $\varepsilon<d, \gamma^{\prime}$ never touches $\gamma^{*}$. Hence, without touching $\gamma^{*}$ we could continuously deform $\gamma^{\prime}$ into a subset of $\gamma$ missing at least one point $Q_{0}$. But any subset 
of $\gamma$ not containing a point of $\gamma$ can be continuously deformed into a point on $\gamma$ such that at any stage of the deformation the set is still on $\gamma$.

If $\gamma^{\prime}$ simply-threads $T_{\varepsilon}(\gamma)$ then it is a continuous deformation of $\gamma$ in $T_{\varepsilon}(\gamma)$ so that $\gamma$ can be continuously deformed into a point such that at every stage of the deformation the curve does not touch $\gamma^{*}$. This contradicts the fact that $\gamma^{*}$ interlocks with $\gamma$, and so Lemma 3.1 is proved for $n=1$.

If $\gamma^{\prime} n$-fold threads $T_{\varepsilon}(\gamma),(n>1)$ we use the fact that $\gamma^{*}$ has the property that the curve $\gamma^{(n)}$ must intersect $\gamma^{*}$ at some stage of a continuous deformation of $\gamma^{(n)}$ into a point. The proof then proceeds as for $n=1$.

In addition to Lemma 3.1 we will also need the following lemma.

LEMMA 3.2. Let $\gamma^{\prime} 2 n$-fold thread $T_{\varepsilon}(\gamma), \gamma$ and $\gamma^{\prime}$ Jordan curves in $R^{3}, \varepsilon>0, n a$ positive integer. For all $\mu>0$ and arbitrary $P$ on $\gamma^{\prime}, \varepsilon$ can be made so small that a second point $P^{\prime}$ can be found on $\gamma^{\prime}$ such that $d\left(P, P^{\prime}\right) \leqq \mu$ with $P P^{\prime} P^{m} P, P P^{\prime \prime} P^{\prime} P$ $n$-fold threading $T_{\varepsilon}(\gamma)$. (See Figure 1 where $n=1$.) The points $P, P^{\prime \prime}, P^{\prime}, P^{\prime \prime}$ occur in that order on $\gamma^{\prime}$.

Proof. Let $\gamma^{*}$ be a Jordan curve interlocking with $\gamma$ and let $d\left(\gamma, \gamma^{*}\right)=\delta$. We first choose $\varepsilon<\delta$ so that $\gamma^{*}$ interlocks with and never touches $\gamma^{\prime}$ at every stage of the continuous deformation of $\gamma^{(n)}$ into $\gamma^{\prime}$.

As $\varepsilon \rightarrow 0$ the $\gamma^{\prime}$ tend uniformly to $\gamma$ and the limiting curve, being a continuous deformation of $\gamma^{\prime}$ which never touches $\gamma^{*}$ at any stage of the deformation, must be a $2 n$-fold covering of $\gamma$ (as is $\gamma^{(2 n)}$.) Any point $Q$ of this limit curve divides it

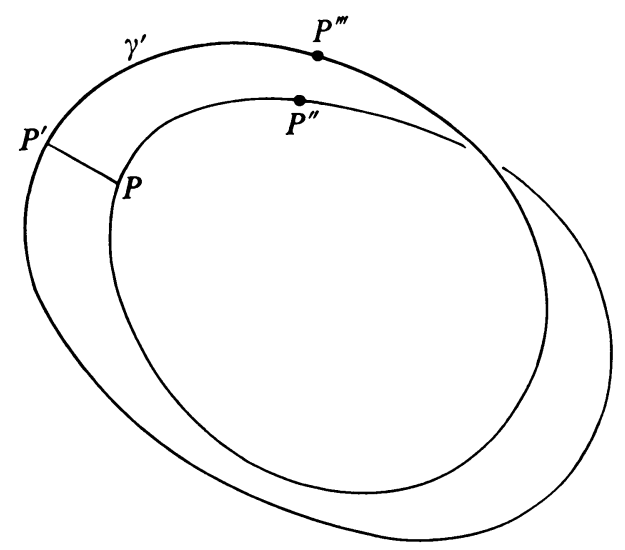

FIGURE 1

into two $n$-fold covering curves. In particular a limit point $Q$ of the $P$ 's will have this property. For $\varepsilon$ sufficiently near zero $Q$ splits into two points on $\gamma^{\prime}, P$ and another point $P^{\prime}$ having, as is easily seen, all the required properties. Lemma 3.2 is therefore proved.

3.2. Doubly-connected minimal surfaces. We proceed to discuss a geometric condition sufficient for the existence of a doubly-connected minimal surface. 
THEOREM 3.1. Let $\gamma$ be a rectifiable Jordan curve of length $L$ in $R^{3}$ and $\eta(\varepsilon)>0$ a function of $\varepsilon>0$ which tends to zero with $\varepsilon$. Then there exists an $\varepsilon_{0}>0$ with the property that whenever $0<\varepsilon \leqq \varepsilon_{0}$, any two Jordan curves $\gamma_{1}, \gamma_{2}$ simply-threading $T_{\varepsilon}(\gamma)$ with lengths $L_{1}, L_{2}$, respectively, satisfying $L_{i} \leqq L+\eta(\varepsilon)$ bound a doublyconnected minimal surface $G ; G$ has area (strictly) smaller than the sum of the minima of areas of disk-like surfaces spanning $\gamma_{1}$ and $\gamma_{2}$ individually.

Proof. The proof presented here is based on the form of the Douglas sufficient condition pertaining to doubly-connected minimal surfaces, i.e., a sufficient condition for the existence of a doubly-connected minimal surface $G$ spanning two rectifiable Jordan curves $\gamma_{1}, \gamma_{2}$ is that the g.l.b. $\delta$, of areas of doubly-connected surfaces spanning $\gamma_{1}, \gamma_{2}$ is (strictly) less than the sum $\sigma$ of the g.l.b.'s of areas of disk-like minimal surfaces spanning $\gamma_{1}$ and $\gamma_{2}$ individually. Also the area $A(G)$ of $G$ is less than $\sigma$.

The present theorem will be proved then if we can prove that the condition of Douglas is satisfied for sufficiently small $\varepsilon$.

The proof will consist of two parts; these are: (1) for each $\varepsilon$ and corresponding $\gamma_{1}$, $\gamma_{2}$ satisfying the conditions of the theorem a special doubly-connected surface $\Delta(\varepsilon)$ spanning $\gamma_{1}, \gamma_{2}$ can be found for which $\lim _{\varepsilon \rightarrow 0} A(\Delta(\varepsilon))=0$, where $A(\Delta)$ represents the area of $\Delta$; (2) as $\varepsilon$ tends to zero the areas of arbitrary disk-like surfaces spanning $\gamma_{1}$ or $\gamma_{2}$ are bounded away from zero. This would imply that $\sigma \geqq \lambda>0$ for all $\varepsilon<\varepsilon^{\prime}$ for some $\lambda>0$ and some $\varepsilon^{\prime}>0$.

From (1) and (2) we would have for any $\varepsilon>0$ satisfying both $\varepsilon<\varepsilon^{\prime}$ and $A(\Delta(\varepsilon))<\lambda$, and for any $\gamma_{1}, \gamma_{2}$ simply-threading $T_{\varepsilon}(\gamma): \delta \leqq A(\Delta(\varepsilon))<\lambda \leqq \sigma$ or $\delta<\sigma$ which is the Douglas sufficient condition. We will have proved the present theorem then if we can verify (1) and (2).

Proof of condition (1). We first describe the surfaces $\Delta=\Delta(\varepsilon)$. To do this we choose some point $O$ on $\gamma$ and an arbitrary $\mu>0$. From Lemma 3.1 we know that if $\varepsilon$ (the radius of $T_{\varepsilon}(\gamma)$ ) is small enough we can find a point $O_{i}$ on $\gamma_{i}$ such that $d\left(O, O_{i}\right) \leqq \mu$. With $O_{i}$ as origin on $\gamma_{i}$ and the same orientation on $\gamma_{i}$ as on $\gamma$ we label points on $\gamma_{i}$ by their arclengths $s_{i}$ measured from $O_{i}: 0 \leqq s_{i}<L_{i}$. Let $P_{i}(\theta)$ be the point on $\gamma_{i}$ with arclength $s_{i}=\theta L_{i}, 0 \leqq \theta<1$. As $\theta$ varies from 0 to $1, P_{i}(\theta)$ starting from $O_{i}$ traverses $\gamma_{i}$ once.

For each $\theta$ join $P_{1}(\theta)$ to $P_{2}(\theta)$ by a straight line segment $P_{1} P_{2}(\theta)$. Then as $\theta$ varies from 0 to $1, P_{1} P_{2}(\theta)$ sweeps out a ruled doubly-connected surface spanning $\gamma_{1}, \gamma_{2}$. We take this surface for $\Delta(\varepsilon)$.

If $l(\theta)$ is the length of $P_{1} P_{2}(\theta)$ then we have

$$
A(\Delta(\varepsilon)) \leqq \max \left(L_{1}, L_{2}\right) \cdot \max _{0 \leqq \theta<1} l(\theta) \leqq[L+\eta(\varepsilon)] \max _{0 \leqq \theta<1} l(\theta) .
$$

We will have verified condition (1) then if we can prove that

$$
\lim _{\varepsilon \rightarrow 0} \max _{0 \leqq \theta<1} l(\theta)=0 .
$$


To prove this we reason indirectly. Suppose that for $\varepsilon_{n} \rightarrow 0$ we can find a sequence of Jordan curves $\gamma_{1 n}, \gamma_{2 n}$ simply-threading $T_{\varepsilon_{n}}(\gamma)$ and $0 \leqq \theta_{n}<1$ for which $L_{i n}$ $\leqq L+\eta\left(\varepsilon_{n}\right)$ and $l_{n}\left(\theta_{n}\right) \geqq \lambda>0$, where $l_{n}\left(\theta_{n}\right)$ is the length of the line segment $P_{1}\left(\theta_{n}\right) P_{2}\left(\theta_{n}\right)$ and $L_{i n}=L\left(\gamma_{i n}\right)$. (We will now drop the subscript $n$ to avoid cumbersome notation.)

From Lemma 3.1 we have $d\left(0, O_{i}\right) \leqq \mu / 2$ where $\mu$ is an arbitrarily small positive number assigned in advance. Applying the triangle inequality we get $d\left(O_{1}, O_{2}\right)$ $\leqq d\left(O, O_{1}\right)+d\left(O, O_{2}\right) \leqq \mu$. Using Lemma 3.1 again and taking $\varepsilon$ smaller if need be we can find a point $Q_{2}$ on $\gamma_{2}$ for which $d\left(Q_{2}, P_{1}\right) \leqq \mu$, for we can first find $P$ on $\gamma$ satisfying $d\left(P, P_{1}\right) \leqq \mu / 2$ and then $Q_{2}$ on $\gamma_{2}$ satisfying $d\left(Q_{2}, P\right) \leqq \mu / 2$.
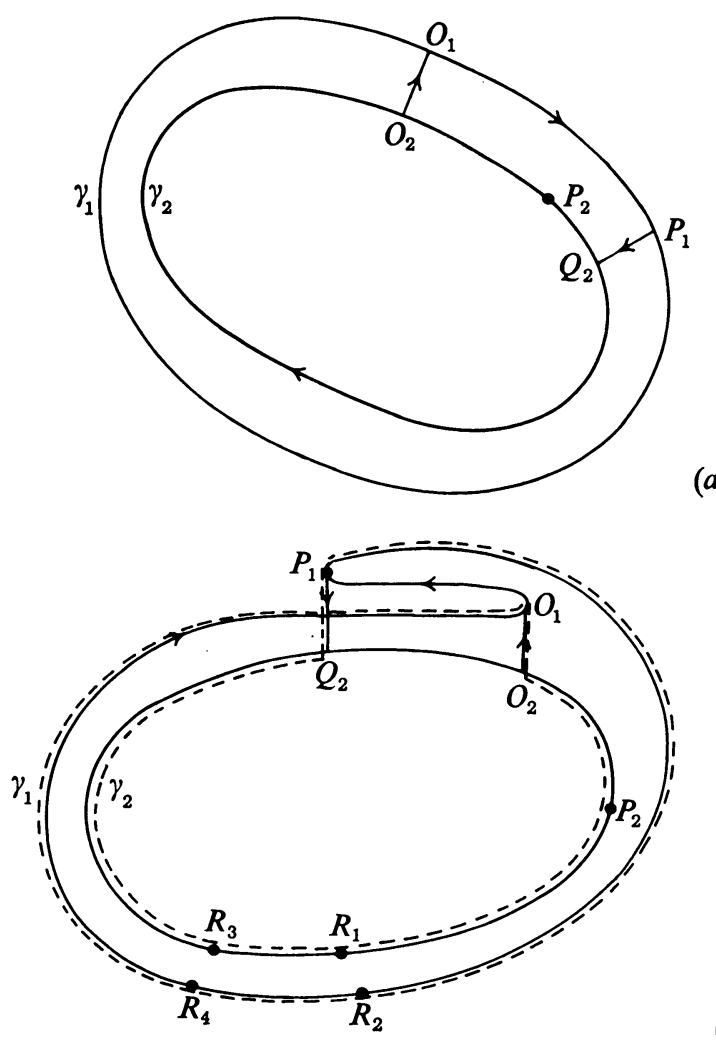

(a)

(b)

FIGURE 2

We shall assume in the following that $\mathrm{O}_{2} \mathrm{P}_{2} Q_{2}$ occur in that order (with respect to arclength on $\gamma_{2}$ ). If the (only) other case $\mathrm{O}_{2} Q_{2} P_{2}$ arises the analysis is similar.

Consider the closed curve $\beta=O_{2} O_{1} P_{1} Q_{2} O_{2}$ where $O_{2} O_{1}, P_{1} Q_{2}$ are line segments and $O_{1} P_{1}, Q_{2} O_{2}$ are arcs of $\gamma_{1}, \gamma_{2}$, respectively, with arclength increasing from $O_{1}$ to $P_{1}$ and from $Q_{2}$ to $O_{2}$. [See Figure 2.]

Two cases can arise:

(a) $\beta$ simply-threads $T_{\mu+\varepsilon}(\gamma)$; (Figure 2a), 
(b) $\beta$ is contractible to a point in $T_{\mu+\varepsilon}(\gamma)$; (Figure 2b).

(The radius $\mu+\varepsilon$ is necessary to ensure that $P_{1} Q_{2}$ lies in the tube.)

Suppose that case (a) arises for an infinite subsequence of $\left\{\varepsilon_{n}\right\}$, which we relabel and take as the whole sequence. Then $\beta=\beta_{n}$ tends uniformly to $\gamma$. From the definition of the length, $L$, of the rectifiable Jordan curve $\gamma$ we have (lower semicontinuity): $\lim _{\inf _{n \rightarrow \infty}} L\left(\beta_{n}\right) \geqq L$. However,

$$
\begin{aligned}
L\left(\beta_{n}\right) & =d\left(O_{2}, O_{1}\right)+L\left(O_{1} P_{1}\right)+d\left(P_{1}, Q_{2}\right)+L\left(Q_{2} O_{2}\right) \\
& \leqq \mu+L\left(O_{1} P_{1}\right)+\mu+L_{2}-L\left(O_{2} Q_{2}\right) \\
& =2 \mu+L_{2}+\left[L\left(O_{1} P_{1}\right)-L\left(O_{2} P_{2}\right)\right]-L\left(P_{2} Q_{2}\right) \\
& \leqq 2 \mu+L_{2}+0-d\left(P_{2}, Q_{2}\right) \\
& \leqq 2 \mu+L_{2}-d\left(P_{1}, P_{2}\right)-d\left(P_{1}, Q_{2}\right) \\
& \leqq 2 \mu+L_{2}-\lambda+\mu \\
& \leqq 3 \mu+\eta(\varepsilon)+L-\lambda .
\end{aligned}
$$

As $\varepsilon \rightarrow 0, \mu \rightarrow 0$ and $\eta(\varepsilon) \rightarrow 0$ so that $\lim _{\inf _{n \rightarrow \infty}} L\left(\beta_{n}\right) \leqq L-\lambda<L$. This contradiction implies that case (a) can arise only a finite number of times. Without loss of generality we suppose that case (b) arises for all $\varepsilon_{n}$.

Case (b) will be disposed of in a similar manner. Consider the curve $\beta^{\prime}=O_{2} Q_{2} P_{1} O_{1} O_{2}$ which doubly-threads $T_{\varepsilon}(\gamma)$ (the dashed curve in Figure $2 \mathrm{~b}$ ). Choosing any point $R_{1}$ on $\beta^{\prime}$ we can find a second point $R_{2}$ on $\beta^{\prime}$ for which $d\left(R_{1}, R_{2}\right) \leqq \mu\left(\lim _{\varepsilon \rightarrow 0} \mu=0\right)$ and $R_{2} R_{1} R_{3} R_{2}, R_{2} R_{4} R_{1} R_{2}$ each simply-thread $T_{\varepsilon}(\gamma)$ (from Lemma 3.2 with $n=1$ ). [See Figure 2b.] $R_{2} R_{1}$ is a line segment and $R_{1} R_{3} R_{2}$, $R_{2} R_{4} R_{1}$ are on $\beta^{\prime}$.

For the length $L\left(\beta^{\prime}\right)$ we have

$$
\begin{aligned}
L\left(\beta^{\prime}\right) & =L\left(O_{1} Q_{2}\right)+d\left(Q_{2}, P_{1}\right)+L\left(P_{1} O_{1}\right)+d\left(O_{1}, O_{2}\right) \\
& \leqq L_{2}-\left[L\left(Q_{2} P_{2}\right)-L\left(O_{2} P_{2}\right)\right]+\mu+\left[L_{1}-L\left(O_{1} P_{1}\right)\right]+\mu \\
& \leqq 2 \mu+L_{1}+L_{2}+\left[L\left(O_{2} P_{2}\right)-L\left(O_{1} P_{1}\right)\right]-L\left(Q_{2} P_{2}\right) \\
& \leqq 2 \mu+L_{1}+L_{2}+0-d\left(Q_{2}, P_{2}\right) \\
& \leqq 2 \mu+L_{1}+L_{2}-\left[d\left(P_{1}, P_{2}\right)-d\left(P_{1}, Q_{2}\right)\right] \\
& \leqq 3 \mu+L_{1}+L_{2}-\lambda .
\end{aligned}
$$

Since $L\left(R_{2} R_{1} R_{3} R_{2}\right)+L\left(R_{2} R_{4} R_{1} R_{2}\right) \leqq L\left(\beta^{\prime}\right)+2 \mu$, we obtain

$$
L\left(R_{2} R_{1} R_{3} R_{2}\right)+L\left(R_{2} R_{4} R_{1} R_{2}\right) \leqq 5 \mu+L_{1}+L_{2}-\lambda .
$$

For one of the curves $R_{2} R_{1} R_{3} R_{2}, R_{2} R_{4} R_{1} R_{2}$, call it $\beta^{*}$, we must have $L\left(\beta^{*}\right)$ $\leqq \frac{1}{2}\left(5 \mu+L_{1}+L_{2}-\lambda\right) \leqq \frac{5}{2} \mu-\frac{1}{2} \lambda+L+\eta(\varepsilon)$ and again we arrive at a contradiction since this last inequality implies that $\lim _{\inf _{n \rightarrow \infty}} L\left(\beta^{*}\right) \leqq L-\frac{1}{2} \lambda<L$ with $\beta^{*} \rightarrow \gamma$ uniformly. Thus, condition (1) is verified. 
Proof of condition (2). We will now show that for some $\lambda>0$ and $\varepsilon^{\prime}>0$ any disk-like surface $\Sigma$ spanning a Jordan curve $\gamma^{\prime}$ simply-threading $T_{\varepsilon}(\gamma)$ with $\varepsilon \leqq \varepsilon^{\prime}$ must have area $A(\Sigma) \geqq \lambda$.

To show this choose some Jordan curve $\gamma^{*}$ interlocking with $\gamma$ with distance $d\left(\gamma^{*}, \gamma\right)=\delta>0$. For $\varepsilon^{\prime}$ we take $\varepsilon^{\prime}=\delta / 3$ and for $\lambda, \lambda=\pi(\delta / 3)^{2}$. That this choice of $\varepsilon^{\prime}$ and $\lambda$ satisfies the requirements can be seen as follows. Let $\Sigma$ be an arbitrary disk-like surface spanning $\gamma^{\prime}$. Consider the tube $T_{\delta / 3}\left(\gamma^{*}\right), \gamma^{*}$ must intersect $\Sigma$. In fact $\gamma^{*}$ translated in any direction a distance not exceeding $\delta / 3$ must also intersect $\Sigma$ since such a translated curve still interlocks with $\gamma$. Consequently, $A(\Sigma)$ $\geqq \pi(\delta / 3)^{2}=\lambda$. Hence condition (2) is verified and Theorem 3.1 is proved.

3.3. Minimal surfaces of the Möbius type. In this paragraph we give a geometric condition sufficient for the existence of minimal surfaces of the Möbius type.

THEOREM 3.2. Let $\gamma$ be a closed, rectifiable Jordan curve of length $L$ in $R^{3}$ and $\eta(\varepsilon)>0$ a function of $\varepsilon>0$ which tends to zero with $\varepsilon$. Then there exists an $\varepsilon_{0}>0$ with the property that whenever $0<\varepsilon \leqq \varepsilon_{0}$ any Jordan curve $\gamma^{\prime}$ doubly-threading $T_{\varepsilon}(\gamma)$ with length $L^{\prime}$ satisfying $L^{\prime} \leqq 2 L+\eta(\varepsilon)$ bounds a minimal surface $G$ of the Möbius type. $G$ has area (strictly) smaller than the g.l.b. of areas of disk-like surfaces spanning $\gamma^{\prime}$.

Proof. The Douglas sufficient condition needed here takes the following form: A sufficient condition for the existence of a minimal surface $G$ of the Möbius type spanning the rectifiable Jordan curve $\gamma^{\prime}$ is that the g.l.b., $\delta$, of areas of Möbius strips spanning $\gamma^{\prime}$ is (strictly) less than the g.l.b., $\sigma$, of areas of disk-like minimal surfaces spanning $\gamma^{\prime}$. $G$ has area less than $\sigma$.

Hence, as in the proof of Theorem 3.1 we need only verify two conditions: (1) for each $\varepsilon$ and corresponding $\gamma^{\prime}$ satisfying the conditions of the theorem a particular Möbius strip $M(\varepsilon)$ spanning $\gamma^{\prime}$ can be found for which $\lim _{\varepsilon \rightarrow 0} A(M(\varepsilon))=0$ where $A(M)$ is the area of $M$, and (2) as $\varepsilon \rightarrow 0$ the areas of arbitrary disk-like surfaces spanning $\gamma^{\prime}$ are bounded away from zero so that $\sigma \geqq \lambda \geqq 0$ for all $\varepsilon \leqq \varepsilon^{\prime}$ for some $\lambda>0$ and some $\varepsilon^{\prime}>0$.

As in the proof of Theorem 3.1 we would then have $\delta \leqq A(M(\varepsilon))<\lambda \leqq \sigma$ for $\varepsilon<\varepsilon^{\prime}$ and $A(M(\varepsilon))<\lambda$, i.e., the Douglas sufficient condition would be satisfied and the theorem proved.

Condition (2) here is proved in the same way as was condition (2) in subsection. 3.2. Except for a small modification the proof of condition (1) here is the same as for condition (1) in paragraph 3.2. We present the modification.

Proof of condition (1). As in 3.2 for $M(\varepsilon)$ we take a ruled surface swept out by a segment $P_{1} P_{2}(\theta)$ with $P_{1}(\theta)$ and $P_{2}(\theta)$ points chosen appropriately on $\gamma^{\prime}$. To describe $P_{i}(\theta)$ we use the result of Lemma 3.2 that for any $\mu>0$ and point $O$ on $\gamma^{\prime}$ if $\varepsilon>0$ is chosen sufficiently small another point $O^{\prime}$ on $\gamma^{\prime}$ can be found such that $d\left(O, O^{\prime}\right) \leqq \mu$ and $O^{\prime} O O^{\prime \prime} O^{\prime}, O^{\prime} O^{\prime \prime} O O^{\prime}$ simply-thread $T_{\varepsilon}(\gamma)$. See Figure 2 with $O, O^{\prime}, O^{\prime \prime}, O^{m}$ replacing $P, P^{\prime}, P^{\prime \prime}, P^{m}$, respectively. 
With orientation on $\gamma^{\prime}$ the same as on $\gamma$ let $P_{1}(\theta)$ be the point on $\gamma^{\prime}$ with arclength $\theta L_{1}$, arclength measured from $O$ as origin; let $P_{2}(\theta)$ be the point on $\gamma^{\prime}$ with arclength $\theta L_{2}$, arclength measured from $O^{\prime}$ as origin. $L_{1}$ is the length of arc $O O^{m} O^{\prime}$, $L_{2}$ the length of $O^{\prime} O^{\prime \prime} O$ (so that $L_{1}+L_{2}=L$ ).

The rest of the proof will be omitted since it follows exactly the reasoning in 3.2 with $O^{\prime} O O^{\prime \prime} O^{\prime}, O^{\prime} O^{\prime \prime} O O^{\prime}$ corresponding to $\gamma_{1}, \gamma_{2}$.

3.4. Generalizations. Theorems 3.1 and 3.2 can easily be generalized to the following

THEOREM 3.3. Let $\gamma$ be a rectifiable Jordan curve in $R^{3}$ with length $L, \eta(\varepsilon)$ a positive function of $\varepsilon$ tending to zero with $\varepsilon$ and $n$ a positive integer. Suppose $\gamma_{1}, \gamma_{2}, \gamma^{\prime}$ to be three rectifiable Jordan curves of lengths $L_{1}, L_{2}, L^{\prime}$, respectively, such that $\gamma_{1}$ and $\gamma_{2}$ $n$-fold thread $T_{\varepsilon}(\gamma)$ while $\gamma^{\prime}, 2 n$-fold threads $T_{\varepsilon}(\gamma)$. Then for some $\varepsilon_{0}>0$, whenever $0<\varepsilon \leqq \varepsilon_{0}$, if $L_{i} \leqq n L+\eta(\varepsilon),(i=1,2)$ and $L^{\prime} \leqq 2 n L+\eta(\varepsilon)$ we can conclude that $\gamma_{1}, \gamma_{2}$ bound a doubly-connected minimal surface $G$ while $\gamma^{\prime}$ bounds a minimal surface $G^{\prime}$ of the Möbius type.

The area $A(G)$ is (strictly) less than the sum of the g.l.b.'s of areas of disk-like surfaces spanning $\gamma_{1}$ and $\gamma_{2}$ individually and the area $A\left(G^{\prime}\right)$ is (strictly) less than the g.l.b. of areas of disk-like surfaces spanning $\gamma^{\prime}$.

The proof of this theorem (not presented here because it has essentially been given in subsections 3.2 and 3.3) is based on Lemmas 3.1 and 3.2.

4. Examples. (1) Let $C_{1}$ be a circle with circumference $2 L$. We suppose that $C_{0}$ is continuously deformed in $R^{3}$ into the double-circle $C_{0}$ and that intermediate positions are denoted by $C_{a}$ with $0<a<1$. Then according to Theorem 3.2 there is a value $a=a_{0}$ such that whenever $0<a \leqq a_{0}, C_{a}$ bounds a minimal surface of the Möbius type.

Also, if $\gamma$ is an arbitrary rectifiable closed Jordan curve in $R^{3}$ and $\gamma_{a}$ congruent to $\gamma$ but displaced a distance $a$ from it, then by Theorem 3.1 there is an $a_{0}>0$ such that whenever $0<a \leqq a_{0}, \gamma, \gamma_{a}$ bound a doubly-connected minimal surface.

(2) Let $\gamma$ and $\gamma_{a}$ be two facing squares of unit edge length, $\gamma_{a}$ displaced a distance $a$ from $\gamma$ in the direction of the normal to the plane of $\gamma$. Then from the Douglas sufficient condition we have that if $a<\frac{1}{2}, \gamma, \gamma_{a}$ bound a doubly-connected minimal surface. From Theorem 2.1 we have that $a>0.6627 \ldots \cdot 2^{1 / 2}=0.9370 \ldots$ implies no doubly-connected minimal surface spans $\gamma, \gamma_{a}$.

It would, of course, be interesting to know that for $\gamma$ any plane curve, a number $a^{*}$ exists for which $0<a \leqq a^{*}$ implies that $\gamma, \gamma_{a}$, bound a doubly-connected minimal surface and for which $a^{*}<a$ implies no doubly-connected minimal surface spans $\gamma$, $\gamma_{a}$ (as in the classical case of coaxial circles).

(3) Let $\gamma_{a}, \gamma_{a}^{\prime}$ be two facing rectangles each with edge lengths 1 and $a$ and separated by the distance $\frac{1}{2}$. The Douglas sufficient condition then tells us that if $1<a, \gamma_{a}, \gamma_{a}^{\prime}$ bound a doubly-connected minimal surface. 
Keeping the distance between $\gamma_{a}$ and $\gamma_{a}^{\prime}$ fixed and decreasing $a$, Theorem 2.3 tells us that there is an $a^{*}>0$ such that if $a<a^{*}$ then no doubly-connected minimal surface spans $\gamma_{a}, \gamma_{a}^{\prime}$. The best (smallest) value of $a^{*}$ to be obtained from Theorem 2.3 we get by minimizing $a=a(c)$ in the relation $1 / 4=c \cosh (a / 2 c)$. The result is $a^{*}=\frac{1}{2} \theta / \cosh \theta$ with $\theta \tanh \theta=1$ or $a^{*}=0.331 \ldots$

\section{BIBLIOGRAPHY}

1. G. A. Bliss, Calculus of variations, Open Court, Chicago, Ill., 1925.

2. R. Courant, Dirichlet's principle, conformal mapping and minimal surfaces, Interscience, New York, 1950.

3. J. Douglas, Solution of the problem of Plateau, Trans. Amer. Math. Soc. 33 (1931), 263321.

4. - The problem of Plateau for two contours, J. Math. Phys. 10 (1931), 315-359.

5. - Some new results in the problem of Plateau, J. Math. Phys. 14 (1936), 55-64.

6. Johannes C. C. Nitsche, A necessary criterion for the existence of certain minimal surfaces, J. Math. Mech. 13 (1964), 659-666.

7. - A supplement to the condition of Douglas, Rend. Circ. Mat. Palermo 13 (1964), 192-198.

THE COOPER UNION,

NeW YoRK, NeW YoRK

Courant Institute of Mathematical Sciences, New York University, New York, New YorK 